\title{
Building complex Kondo impurities by manipulating entangled spin chains
}

Deung-Jang Choi ${ }^{1,2, \$}$, Roberto Robles ${ }^{3}$, Shichao Yan ${ }^{1,2, \$}$, Jacob A. J. Burgess ${ }^{1,2, \uparrow}$, Steffen RolfPissarczyk $^{1,2}$, Jean-Pierre Gauyacq ${ }^{4}$, Nicolás Lorente ${ }^{5,6}$, Markus Ternes ${ }^{2}$, Sebastian Loth ${ }^{1,2,7}$

1 Max Planck Institute for the Structure and Dynamics of Matter, Luruper Chaussee 149, 22761 Hamburg, Germany

2 Max Planck Institute for Solid State Research, Heisenbergstr. 1, 70569 Stuttgart, Germany

3 Catalan Institute of Nanoscience and Nanotechnology (ICN2), CSIC and The Barcelona

Institute of Science and Technology, Campus UAB, Bellaterra, 08193 Barcelona, Spain

4 Institut des Sciences Moléculaires d'Orsay (ISMO), CNRS, Univ. Paris-Sud, Université ParisSaclay, Bât.351, 91405 Orsay Cedex, France

5 Centro de Física de Materiales, CFM/MPC (CSIC-UPV/EHU), Paseo Manuel de Lardizabal 5, 20018 Donostia-San Sebastián, Spain

6 Donostia International Physics Center (DIPC), Paseo Manuel de Lardizabal 4, 20018 Donostia-San Sebastián, Spain

7 Institute for Functional Matter and Quantum Technologies, University of Stuttgart, Pfaffenwaldring 57, 70569 Stuttgart, Germany

$\S$ Present address: CIC nanoGUNE, Tolosa Hiribidea 78, Donostia-San Sebastián 20018, Spain

$\$$ Present address: School of Physical Science and Technology, ShanghaiTech University, Shanghai 201210, China,

+ Present address: Department of Physics and Astronomy, University of Manitoba, Winnipeg, Manitoba R3T 2N2, Canada

The creation of molecule-like structures in which magnetic atoms interact controllably is full of potential for the study of complex or strongly correlated systems. Here, we create spin chains in which a strongly correlated Kondo state emerges from magnetic coupling of transition-metal atoms. We build chains up to ten atoms in length by placing Fe and Mn atoms on a $\mathrm{Cu}_{2} \mathrm{~N}$ surface with a scanning tunneling microscope. The atoms couple antiferromagnetically via super-exchange interaction through the nitrogen atom network of the surface. The emergent Kondo resonance is spatially distributed along the chain. Its strength can be controlled by mixing atoms of different transition metal elements and manipulating their spatial distribution. We show that the Kondo screening of the full chain by the electrons of the non-magnetic substrate depends on the inter-atomic entanglement of the spins in the chain, demonstrating the prerequisites to build and probe spatially extended strongly correlated nanostructures.

Keywords: Kondo effect, Spin chains, Entanglement, Scanning Tunneling Microscopy

This document is the Accepted Manuscript version of a Published Work that appeared in final form in Nano Letters, copyright $(\mathcal{C}$ American Chemical Society after peer review and technical editing by the publisher. To access the final edited and published work see: https://dx.doi.org/10.1021/acs.nanolett.7b02882 
Conduction electrons scattering off a magnetic impurity can screen the localized magnetic moment by forming a non-magnetic many-particle singlet state. This is known as the Kondo effect ${ }^{1}$. On a metallic surface, Kondo screening of a magnetic moment manifests itself as a prominent zero-bias resonance in scanning tunneling microscope (STM) differential conductance measurements ${ }^{2}$. Ample experimental evidence exists for Kondo resonances of single spins on metals, either atomic or molecular ${ }^{2-6}$. However, an entirely different world is revealed when several spins interact on metallic hosts ${ }^{7-11}$. An extensive body of theoretical work explores the rich range of new states of matter that can be created from the competition of Kondo screening and inter-spin interactions ${ }^{12-17}$. Such quantum many-body phenomena exhibit extraordinary complexity but offer great potential if the emergent quantum states can be controlled ${ }^{18,19}$. The Kondo effect in spin-coupled chains, has been proposed as a means of transmitting quantum coherent information in solid-state environments ${ }^{20}$. This concept requires ways to extend ${ }^{21,22}$ and manipulate $3,23,24$ the Kondo effect on the atomic level. To date, in most scenarios considered experimentally, each spin is individually Kondo-screened and the interaction among spins drives the pre-existing Kondo effect into a new phase of matter ${ }^{8,22,25}$. These Kondo chains and lattices are keystones in understanding heavy-fermion compound ${ }^{26,27}$, entanglement in condensed matter $^{28}$ or in transmitting information quantum coherently through chains of spins ${ }^{20,29,30}$. However, the intriguing possibility to create emergent Kondo states in nanostructure systems built from atoms that, individually, are not Kondo screened is unexplored.

Using the atom manipulation capabilities of the STM, we assemble one-dimensional chains of atoms composed of $\mathrm{Fe}$ and $\mathrm{Mn}$ atoms on a monolayer film of copper nitride, $\mathrm{Cu}_{2} \mathrm{~N}$, grown on $\mathrm{Cu}$ (100). We use a low-temperature STM to assemble chains of up to ten atoms in length, Fig. 1a,c. We find that chains with one Fe and an odd number of Mn atoms exhibit a collective Kondo state. The Kondo state in the composite system appears only if the spin-spin interactions within the chain are engineered to entangle all atomic spins with each other and form a doubly degenerate ground state that enables electrons from the host metal to flip all spins in the chain by a single electron scattering event. Atomically precise construction allows exact determination of the chain composition and tuning of coupling strengths. This in turn determines the degree of inter-atomic entanglement which influences the probability of spin-flip scattering and the resulting efficiency of the Kondo screening. Thus the control we have over atomic entanglement in the atom chain allows us to create and tune the emergence of a many-body electronic state.

The magnetic moments of $\mathrm{Fe}$ and $\mathrm{Mn}$ atoms on the $\mathrm{Cu}$ binding site of $\mathrm{Cu}_{2} \mathrm{~N}$ are well-described as spins with magnitude $2(\mathrm{Fe})$ and $5 / 2(\mathrm{Mn})^{7,31}$. Due to their easy axis magnetic anisotropy, neither Fe nor Mn forms a Kondo state individually at the temperature of our experiment, $T=0.5 \mathrm{~K}^{4,32}$. 
Spectra of the differential conductance, $\mathrm{d} I / \mathrm{d} V(V)$, recorded with the tip positioned over each atom show spin excitations at finite energy but no Kondo resonance at zero bias (Fig. 1b).

Surprisingly, the two-atom chain, one $\mathrm{Mn}$ and one $\mathrm{Fe}$ atom, shows a drastically altered conductance spectrum, Fig. 1b. Spin excitations appear at $\pm 16 \mathrm{mV}$ and $\pm 24 \mathrm{mV}$ bias and a clear resonance is visible at zero bias. The spectra show spin excitations compatible only with an antiferromagnetic coupling of the atomic magnetic moments as in $\mathrm{Mn}$ dimers $^{7}$ or Fe dimers ${ }^{33}$. Density functional theory calculations (DFT) confirm the antiferromagnetic order within the chain and show that the atomic magnetic moments stay isolated ${ }^{34}$, Fig. 1d (see also Supplementary Fig. S3).

The zero-bias resonance on $\mathrm{MnFe}$ shows the behaviour expected for a Kondo resonance: the peak can be fit by a Frota function with a width of $\Gamma_{K}{ }^{0}=1.47 \pm 0.05 \mathrm{meV}$ (see Supplementary Fig. S4 for fit details) corresponding to a Kondo temperature of $T_{\mathrm{K}}=17 \pm 1 \mathrm{~K}$, (Fig. 1b). It quickly broadens with increasing temperature and splits in magnetic field confirming its magnetic many-body nature (Supplementary Fig. S1).

To test whether this zero-bias resonance is a robust effect related to the chain's spin ground state rather than properties of the constituent atoms we assembled chains of the type $\mathrm{Mn}_{\mathrm{x}} \mathrm{Fe}$ with $\mathrm{x}=1, \ldots, 10$ (Fig. 2a,b). We find that the presence of Kondo scattering strictly depends on the parity of the chain: all chains with an odd number of Mn atoms show a similar zero-bias resonance (Fig. 2a), whereas chains with an even number of Mn atoms do not (Fig. 2b).

To elucidate the origin of the Kondo resonance in the $\mathrm{Mn}_{\mathrm{x}} \mathrm{Fe}$ chains we use an effective spin Hamiltonian that includes antiferromagnetic Heisenberg-type exchange interaction, $J$, between $\mathrm{Mn}$ and $\mathrm{Fe}$ atoms, $J^{\prime}$, between nearest neighbour Mn atoms and easy-axis magnetic anisotropy of the Fe atom, $D$ (see Supplementary Section $\mathrm{S} 1$ for details). $J, J$ ' and $D$ are determined by fitting the experimental $\mathrm{d} I / \mathrm{d} V$ spectra and a single set of model parameters achieves good fitting for all chains: $J=(13.3 \pm 1.0) \mathrm{meV}, \quad J^{\prime}=(4.2 \pm 0.8) \mathrm{meV}$, and $D=(4.0 \pm 0.4) \mathrm{meV}$ (Supplementary Fig. S2). Inclusion of other terms such as transverse anisotropy of $\mathrm{Fe}$, or anisotropy of the $\mathrm{Mn}$ atom do not change the results significantly and were left out to keep the number of fit parameters minimal. We calculate the conductance spectra with a perturbative approach which accounts for spin-flip scattering processes up to $3^{\text {rd }}$ order in the interaction matrix elements because this $3^{\text {rd }}$ order perturbation approach correctly captures the effect of Fermi-surface anomalies on tunneling and contains the logarithmic Kondo peak. ${ }^{35}$ This method reproduces the essential properties of Kondo resonances both in atomic and molecular systems ${ }^{5}$. The simulated 
spectra reproduce the spin excitations in all $\mathrm{Mn}_{\mathrm{x}} \mathrm{Fe}$ chains and also reproduce the appearance of Kondo resonances exclusively in chains with an odd number of $\mathrm{Mn}$ atoms (Fig. 2c, Supplementary Fig. S6).

Inspection of the spin wave functions of the $\mathrm{Mn}_{(2 \mathrm{n}+1)} \mathrm{Fe}$ atomic chains shows that the spin ground states of all chains are in a low total spin configuration due to the antiferromagnetic coupling (see also Supplementary Fig. S3) and doubly degenerate. The presence of a doubly degenerate ground state alone, however, is not sufficient to form the many-electron Kondo resonance. Kondo phenomena emerge when the scattering from a substrate electron induces switching between these degenerate ground states ${ }^{5,36}$.

Hence, the Kondo resonances found for the $\mathrm{Mn}_{(2 \mathrm{n}+1)} \mathrm{Fe}$ chains are peculiar. The underlying Kondo scattering has no classical analogue as it involves the spin flip of up to 10 magnetic atoms $\left(\mathrm{Mn}_{9} \mathrm{Fe}\right.$ case) with spin magnitudes $5 / 2$ and 2 by only one spin $1 / 2$ electron. It must therefore rely on the presence of superposition spin states within the spin chain that have contributions of all basis states of all atoms and thus permit the classically forbidden transition. Antiferromagnetic Heisenberg-type exchange coupling as present in our atom chains is known to create such superposition states through entanglement of the constituent $\operatorname{spins}^{30}$.

The importance of superposition spin states is illustrated in comparing the complementary chains $\mathrm{Mn}_{3} \mathrm{Fe}$ and $\mathrm{MnFe}_{3}$ (Fig. 2c,d). Both spin chains show similar spin excitation spectra at approximately $\pm 10 \mathrm{mV}$ and have doubly degenerate ground states. But only $\mathrm{Mn}_{3} \mathrm{Fe}$ has a pronounced Kondo resonance at zero bias. The key difference between $\mathrm{MnFe}_{3}$ and $\mathrm{Mn}_{3} \mathrm{Fe}$ is in the composition of the spin ground states (Fig. 2 e, g). In $\mathrm{MnFe}_{3}$ the presence of three Fe atoms with large anisotropy, $D$, creates a ground state that has a strong Néel state character. Each atom has a well-defined orientation of the spin: projecting one of the ground state wave functions onto the basis states of each atom shows that the Mn atom's spin is mostly $+5 / 2$ and the Fe atoms alternate between -2 or +2 (Fig. $2 \mathrm{~g}$ ). The atomic spins are correlated with each other but not in superposition states, so that an electron scattering on one of the atoms cannot flip the other atomic spins in the chain strongly reducing the probability for scattering between the two degenerate ground states (Fig. 2f) and prohibiting the formation of a Kondo singlet state ${ }^{37}$. By contrast, in $\mathrm{Mn}_{3} \mathrm{Fe}$ the vanishing axial anisotropy of the Mn atoms leads to two ground states that are superposition states with significant contributions from many basis states of each atom (Fig. 2e). The atomic spins are entangled and a single electron scattering on one of the atomic spins can flip the whole spin chain between its two ground states with significant probability permitting the formation of a collective Kondo resonance (Fig. 2e). 
The relation between entanglement and strength of the Kondo resonance is substantiated by investigating $\mathrm{MnFe}$ chains where the ratio between the inter-atomic exchange coupling strength, $J$, and the magnetic anisotropy energy, $D$, can be adjusted by varying the separation between the atoms (Fig. 3a). We record the amplitude of the Kondo resonance in the $\mathrm{d} I / \mathrm{d} V$ spectra of each chain (Fig. 3c) and use the spin excitations at finite energy to extract $J /|D|$ for each chain built. We find that chains with $J /|D|<<1$ show no Kondo resonance. Strongly-coupled FeMn chains with $J /|D| \gg>1$ exhibit prominent Kondo resonances whereby their amplitude increases with increasing $J /|D|$. Since MnFe is a bipartite spin system, the von Neumann mutual information of the Fe and Mn spin subsystems serves as a measure of entanglement (Fig. 3b, see Methods), and we extract it from the spin Hamiltonian model by inputting $J /|D|$. MnFe chains with small $J /|D|$ have minimal entanglement. In such chains uniaxial anisotropy of Fe dominates and drives the chain into Néel-like ground states without entanglement (Fig. 3d). Increasing $J /|D|$ increases entanglement between the Fe and $\mathrm{Mn}$ atoms as seen by increasing von Neumann entropy. This drives the chain into superposition spin states that enable Kondo screening (Fig. 3e).

A Kondo resonance in the $\mathrm{Mn}_{\mathrm{x}} \mathrm{Fe}$ chains is not compatible with a single electron in a molecular orbital spanning the chains. This type of molecular Kondo effect would not follow the observed even-odd effect of Kondo resonances being present only on chains with $\mathrm{x}=1,3, \ldots$ Moreover, our DFT calculations find that all magnetic moments of the chain are localized in the $d$-orbitals of the Fe and $\mathrm{Mn}$ atoms ${ }^{34}$ (Supplementary Fig. S3). No spin-polarized molecular state can be identified that could enable Kondo screening through reversible electron transfer from the metal to a hybridized orbital of the full chain. This makes it possible to resolve the spatial structure of the Kondo resonance within the spin chains.

Conductance spectra acquired along the $\mathrm{Mn}_{\mathrm{x}} \mathrm{Fe}$ chains, $\mathrm{d} I / \mathrm{d} V(V, d)$, show that the Kondo resonance is delocalized (Fig. $4 \mathrm{a}$ ). However, the entanglement among the atoms in the chain does not lead to a structureless magnetic object as could be expected for a macrospin. Contrarily, the resonance increases along the chain and is strongest at the $\mathrm{Mn}$ atom furthest from the $\mathrm{Fe}$ atom. This is surprising because the Fe atom is required for the emergence of the Kondo resonance and emphasizes that, even for the longest chain $\left(\mathrm{Mn}_{9} \mathrm{Fe}\right)$, it must be caused by collective screening of entangled spin states of the entire chain.

Remarkably, the maximum amplitude of the Kondo resonance increases approximately linearly with chain length. For $\mathrm{Mn}_{9} \mathrm{Fe}$ it reaches up to $80 \%$ of the amplitude of the spin-excitationinduced conductance steps (Fig. 4c). This behaviour is consistent with an increased efficiency of 
electron-spin scattering between the two degenerate ground states by substrate electrons. The increased number of scattering sites provided by longer chains increases the interaction between the spin chain and the electron bath. In addition, the Fe atom's magnetic anisotropy becomes effectively diluted with increasing number of $\mathrm{Mn}$ atoms allowing for a more efficient Kondo screening. Therefore length, geometry and composition of the chain are tunable parameters that permit us to vary the degree of entanglement of the atoms in the chain and as a consequence, the Kondo state.

These measurements demonstrate that it is experimentally feasible to create a Kondo ground state within a chain of atoms with large magnetic moments. The emergent Kondo state can be tuned by a competition between inter-atomic spin interaction and magneto-crystalline anisotropy of the constituent atoms. The Kondo state thus depends on the specific atomic composition of the spin chain and is characterized by the degree of entanglement among the atoms of the chain. This indicates that surface-adsorbed spin chains can serve as prototype systems for the exploration of correlated condensed-matter phases, where the electron correlations are tailored by the specific design of the atomic chains. In long chains ( $x$ »10) it may be possible to study the impact of decoherence on the correlated states. Beyond testing prominent theoretical Kondo chain and lattice models ${ }^{13,16}$ this method can in principle be generalized to a broader class of materials where electron-electron interaction can be tuned by structure and composition.

\section{Methods}

Experimental parameters. All measurements used a low-temperature ultrahigh vacuum scanning tunnelling microscope (Unisoku USM 1300 3He) with base temperature of $0.5 \mathrm{~K}$. A clean $\mathrm{Cu}(100)$ surface with large monatomically flat terraces was prepared by repeated Ar sputtering and annealing to $850 \mathrm{~K}$. Then, a monolayer of copper nitride, $\mathrm{Cu}_{2} \mathrm{~N}$, was formed as a decoupling layer by sputtering with $\mathrm{N}_{2}$ at $1 \mathrm{kV}$ and annealing to $600 \mathrm{~K}$. Fe and $\mathrm{Mn}$ atoms were deposited onto the precooled surface by thermal evaporation of elemental Fe and $\mathrm{Mn}$ from effusion cells. All atom chains were created by vertical atom manipulation ${ }^{7}$. Prior to manipulation $\mathrm{Fe}$ and $\mathrm{Mn}$ atoms were identified using their characteristic conductance spectra ${ }^{31}$. Differential conductance spectra, $\mathrm{d} I / \mathrm{d} V(V)$, record the differential conductance of the tunnel junction as a function of sample bias detected by Lock-In detection of the tunnelling current caused by adding a modulation voltage of $72 \mu \mathrm{V}_{\text {rms }}$ amplitude at $691 \mathrm{~Hz}$ to the bias. 
Model spin Hamiltonian parameters. The model applied to the spin chains uses an effective spin Hamiltonian to describe the spin states of the $\mathrm{Mn}_{\mathrm{x}} \mathrm{Fe}$. Conductance spectra are calculated by considering electron-spin scattering with tunnelling electrons (Supplementary Figure S2).

The spin states of the $\mathrm{MnFe}$ chains represented by their density matrices, $\rho$, are completely determined by the ratio $J /|D|$ (Fig. 2d). We quantify the degree of entanglement for the dimers (Fig. $2 \mathrm{fg}$ ) by entanglement entropy ${ }^{38}, I(\rho)$. For this analysis we add an infinitesimal magnetic field to the spin Hamiltonian and set temperature to zero to ensure that $\rho$ is a pure state. $I(\rho)$ is obtained by calculating the von Neumann mutual information of the $\mathrm{Fe}$ and $\mathrm{Mn}$ spin subsystems, $I(\rho)=S\left(\rho_{\mathrm{Mn}}\right)+S\left(\rho_{\mathrm{Fe}}\right)-S(\rho) \quad$ in the basis that minimizes $I(\rho)$ (see Supplementary Fig. S5). We find that this basis coincides with the coordinate system determined by the magnetic anisotropy axis of the Fe atom. $S$ is the von Neumann entropy and $\rho_{\mathrm{Fe}}$ and $\rho_{\mathrm{Mn}}$ are the reduced density matrices of the $\mathrm{Fe}$ and $\mathrm{Mn}$ spin obtained by partial trace over $\rho$. Since the spins considered here are larger than $1 / 2$ we normalised $I(\rho)$ to the entanglement entropy of the maximally entangled state, so that $I(\rho)$ ranges from 0 for chains where Fe and Mn are not correlated or only classically correlated $^{39}$ to 1 for chains where Fe and Mn are maximally entangled.

Density function theory calculations. Collinear DFT $+U$ calculations were performed with the VASP code, using the PBE xc functional together with charging-energy corrections in the Dudarev et al. scheme (See Supplementary Section S2 for details). The d electrons for Fe and Mn had an onsite energy of $U-J=1 \mathrm{eV}$ and $4.0 \mathrm{eV}$ respectively. For all chains we find the magnetic ground state of the $\mathrm{Mn}_{\mathrm{x}} \mathrm{Fe}$ chains to be close to $m_{\mathrm{T}}=1 / 2$ due to antiferromagnetic ordering of the Fe and Mn magnetic moments.

\section{Supporting Information.}

Supplementary Section S1: Spin Hamiltonian model and calculation of the differential conductance spectra. Supplementary Section S2: Details on the Density Function calculations of $\mathrm{FeMn}_{\mathrm{x}}$ chains. Supplementary Figures S1 to S6. Supplementary References. 


\section{FIGURE CAPTIONS}

Figure 1| Emergence of Kondo screening in $\mathrm{Mn}_{\mathbf{x}} \mathrm{Fe}$ spin chains. a, Ball model and STM images of the construction of a MnFe dimer by adding a Mn atom (green) to an Fe atom (red) on the $\mathrm{Cu}_{2} \mathrm{~N}$ surface $(\mathrm{Cu}$ atoms yellow, $\mathrm{N}$ atoms blue). b, Differential conductance spectra, $\mathrm{d} I / \mathrm{d} V(V)$, for a $\mathrm{Mn}$ atom, a Fe atom and a $\mathrm{MnFe}$ chain. $\mathrm{MnFe}$ shows a prominent Kondo resonance at $0 \mathrm{~V}$ that is fit well by a Frota lineshape with a half-width at half-maximum, $\Gamma_{\mathrm{K}}=1.47 \mathrm{meV}$ (see Supplementary Fig. S4 for fit details). c, Ball model and STM image of $\mathrm{Mn}_{9} \mathrm{Fe}$ spin chain after it was constructed from $\mathrm{MnFe}$ in (a) by adding 8 additional Mn atoms. d, Spin density distribution on $\mathrm{Mn}_{9} \mathrm{Fe}$ chain calculated by density function theory (see Methods) shown as density difference between spin-up (blue) and spin-down (red) states. Isosurface value $0.05 \mathrm{e} / \AA^{3}$. Copper atoms (yellow) and Nitrogen atoms (cyan). STM image sizes are $(5 \times 5) \mathrm{nm}^{2}$ in (a) and $(7 \times 5) \mathrm{nm}^{2}$ in (c) shown as $3 \mathrm{~d}$ rendering with height from low ( $0 \AA$, orange) to high (4 Å, white). Tunnel junction setpoint $10 \mathrm{mV}$ and $100 \mathrm{pA}$.

Figure 2| Dependence of Kondo screening on chain composition. a, Differential conductance spectra, $\mathrm{d} I / \mathrm{d} V(V)$, of $\mathrm{Mn}_{(2 \mathrm{n}+1)} \mathrm{Fe}$ chains with an odd number of $\mathrm{Mn}$ atoms in the chain showing Kondo resonances at zero bias. b, $\mathrm{d} I / \mathrm{d} V$ spectra of $\mathrm{Mn}_{(2 \mathrm{n})} \mathrm{Fe}$ chains with an even number of $\mathrm{Mn}$ atoms in the chain showing no Kondo resonances. c, Measured (purple) and calculated (blue) spectra of $\mathrm{Mn}_{3} \mathrm{Fe}$ showing a prominent Kondo resonance at $0 \mathrm{~V}$ (black arrow). d, Measured (light purple) and calculated (blue) spectra of $\mathrm{MnFe}_{3}$ showing no Kondo resonance. Calculation includes nearest neighbour spin coupling between Fe atoms, $J^{\prime \prime}=11.0 \mathrm{meV}$. e, Spin wave function composition of one of the two ground states, $\psi_{1}$, for $\mathrm{Mn}_{3} \mathrm{Fe}$ plotted as the projection of the reduced wave function for the i-th atom, $\left|\left\langle m_{\mathrm{i}} \mid \psi_{1}\right\rangle_{\mathrm{i}}\right|$, onto the basis states, $\left|m_{\mathrm{i}}\right\rangle$, chosen as the eigenstates of the $S_{\mathrm{i}, \mathrm{z}}$ operator. The ground state has contributions of several $\left|m_{\mathrm{i}}\right\rangle$ states on each atom enabling the large transition intensity seen in the middle plot. Colour shows magnitude from 0 (white) to 1 (green). The ball model shows the atomic arrangement of $\mathrm{Mn}_{3} \mathrm{Fe}(i=1$ is the leftmost Mn atom). f, Transition intensity between the two degenerate ground states $\psi_{1}, \psi_{2}$ of $\mathrm{Mn}_{3} \mathrm{Fe}$ (purple line) and $\mathrm{MnFe}_{3}$ (pink line) evaluated as the transition matrix element of the

operator $\vec{S}_{i} \vec{\sigma}$ for each atom, $i$, of the chain. $\mathrm{Mn}_{3} \mathrm{Fe}$ has a large transition intensity for single electron scattering making Kondo screening possible, whereas $\mathrm{MnFe}_{3}$ has negligible transition intensity. $\mathbf{g}$, Spin wave function composition of of one of the two ground states, $\psi_{1}$, for $\mathrm{MnFe}_{3}$ plotted analogous to (e) and a ball model showing the atomic arrangement ( $i=1$ is the Mn atom). The ground state of $\mathrm{MnFe}_{3}$ is composed mostly of one $\left|m_{\mathrm{i}}\right\rangle$ state on each atom resulting in negligible transition intensity. 
Figure 3| Dependence of Kondo screening on spin coupling strength. a, STM images of MnFe chains with varying separation: A: 2 unit cells $(0.72 \mathrm{~nm}), \mathrm{B}: 1$ unit cell $(0.36 \mathrm{~nm}), \mathrm{C}$ : compact $(0.3 \mathrm{~nm})$. Image size $(3.4 \times 2.3) \mathrm{nm}^{2}$ shown as $3 \mathrm{D}$ rendering of height with color from low (orange) to high (white). b, Entanglement entropy, $I$, scaled to the entropy of a maximally entangled chain, $I_{\max }$, plotted as function of effective coupling strength, $J /|D|$, of the chain (see Methods for details). c, Relative Kondo peak amplitude, $A_{\mathrm{K}} / A_{\text {iet }}$, as a function of $J /|D|$. The amplitudes of the Kondo resonance, $A_{\mathrm{K}}$, and spin excitations, $A_{\text {iet, }}$ and $J /|D|$ were extracted by fitting $\mathrm{d} I / \mathrm{d} V(V)$ spectra of each chain (see Supplementary Fig. S4). d, Left panel : $\mathrm{d} I / \mathrm{d} V(V)$ spectrum of $\mathrm{MnFe}$ chain A measured on the $\mathrm{Mn}$ atom position. A spin excitation at $1 \mathrm{mV}$ is visible but no Kondo resonance at $0 \mathrm{~V}$. Right panel: composition of MnFe chain A's ground state spin wave function (plotted in the same manner as Fig. 2e, g). e, Left panel : $\mathrm{d} I / \mathrm{d} V(V)$ spectrum of $\mathrm{MnFe}$ chain $\mathrm{C}$ showing a prominent Kondo resonance. Right panel: ground state spin wave function of $\mathrm{MnFe}$ chain $\mathrm{C}$.

Figure 4| Spatial evolution of Kondo screening in $\mathbf{M n}_{\mathbf{x}} \mathrm{Fe}$ chains. a, Maps of the differential conductance, $\mathrm{d} I / \mathrm{d} V(V, d)$, measured along the $\mathrm{Mn}_{\mathrm{x}} \mathrm{Fe}$ spin chains and plotted colour-coded as a function of sample bias, $V$, and displacement, $d$, along the spin chains. Atom positions are indicated by red $(\mathrm{Fe})$ and green $(\mathrm{Mn})$ dashes. The first $\mathrm{Mn}$ atom of each chain equals $\mathrm{d}=0$. Ball model of $\mathrm{Mn}_{9} \mathrm{Fe}$ is shown for reference with lateral scale matched to the $\mathrm{d} I / \mathrm{d} V(V, d)$ spectra. The delocalized Kondo resonance is visible as horizontal yellow streak centred at $0 \mathrm{~V}$ bias. b. Spatial profile of the relative Kondo peak amplitude, $A_{\mathrm{K}} / A_{\text {iet }}$, along each spin chain from $\mathrm{MnFe}$ (orange points) to $\mathrm{Mn}_{9} \mathrm{Fe}$ (black points). The Kondo resonance drops off at approximately $1.5 \mathrm{~nm}$ distance from the edge $\mathrm{Mn}$ atom in the longer chains $(x=5,7,9)$. c, Evolution of $A_{\mathrm{K}} / A_{\text {iet, }}$ with chain length evaluated at the position of the first $\mathrm{Mn}$ atom $(\mathrm{d}=0 \mathrm{~nm})$ showing a linear increase of Kondo resonance amplitude with chain length. 


\section{REFERENCES}

1. Kondo, J.; Progress of Theoretical Physics 1964, 32, 37.

2. Madhavan, V.; Chen, W., Jamneala, T.; Crommie, M. F.; Wingreen, N. S. Science 1998, 280, 567-569.

3. Zhao, A.; Li, Q.; Chen, L.; Xiang, H.; Wang, W.; Pan, S.; Wang, B.; Xiao, X.; Yang, J.; Hou, J. G.; Zhu, Q. Science 2005, 309, 1542-1544.

4. Otte, A. F.; Ternes, M.; von Bergmann, K.; Loth, S.; Brune, H.; Lutz, C. P.; Hirjibehedin, C. F.; Heinrich, A. J. Nat. Phys. 2008, 4, 847.

5. Ternes, M. New Journal of Physics 2015, 17, 063016.

6. von Bergmann, K.; Ternes, M.; Loth, S.; Lutz, C. P.; Heinrich, A. J. Phys. Rev. Lett. 2015, 114, 076601.

7. Hirjibehedin, C. F.; Lutz, C. P.; Heinrich, A. J. Science 312(5776), 1021-1024 May (2006).

8. Toskovic, R.; van den Berg, R.; Spinelli, A.; Eliens, I. S.; van den Toorn, B.; Bryant, B.; Caux, J.-S.; Otte, A. F. Nat Phys 2016, 12, 656-660.

9. Otte, A. F.; Ternes, M.; Loth, S.; Lutz, C. P.; Hirjibehedin, C. F.; Heinrich, A. J. Phys. Rev. Lett. 2009, 103, 107203.

10. Segovia, P.; Purdie, D.; Hengsberger, M.; Baer, Y. Nature 1999, 402, 504-507.

11. Yan, S.; Choi, D.-J.; Burgess, J. A. J.; Rolf-Pissarczyk, S.; Loth, S. Nat Nano 2015, 10, 4045.

12. Affleck, I.; Ludwig, A. W. W.; Jones, B. A. Phys. Rev. B 1995, 52, 9528-9546.

13. Tsvelik, A. M. Yevtushenko, O. M. Phys. Rev. Lett. 2015, 115, 216402.

14. Nozières, P. J. Phys. Soc. Jpn. 2005, 74, 4-7.

15. Jones, B. A.; Varma, C. M.; Wilkins, J. W. Phys. Rev. Lett. 1988, 61, 125-128.

16. Saguia, A. Sarandy, M. S. Phys. Rev. A 2003, 67, 012315.

17. Lobos, A. M.; Cazalilla, M. A.; Chudzinski, P. Phys. Rev. B 2012, 86, 035455.

18. Dagotto, E. Science 2005, 309, 257-262.

19. Takagi, H. Hwang, H. Y. Science 2010, 327, 1601-1602.

20. Bayat, A.; Bose, S.; Sodano, P.; Johannesson, H. Phys. Rev. Lett. 2012, 109, 066403.

21. Jiang, Y.; Zhang, Y. N.; Cao, J. X.; Wu, R. Q.; Ho, W. Science 2011, 333, 324-328.

22. DiLullo, A.; Chang, S.-H.; Baadji, N.; Clark, K.; Klöckner, J.-P.; Prosenc, M.-H.; Sanvito, S.; Wiesendanger, R.; Hoffmann, G.; Hla, S.-W. Nano Letters 2012, 12, 3174-3179.

23. Oberg, J. C.; Calvo, M. R.; Delgado, F.; Moro-Lagares, M.; Serrate, D.; Jacob, D.; Fernandez-Rossier, J.; Hirjibehedin, C. F. Nat Nano 2014, 9, 64-68.

24. Parks, J. J.; Champagne, A. R.; Costi, T. A.; Shum, W. W.; Pasupathy, A. N.; Neuscamman, E.; Flores-Torres, S.; Cornaglia, P. S.; Aligia, A. A.; Balseiro, C. A.; Chan, G. K.-L.; Abruna, H. D.; Ralph, D. C. Science 2010, 328, 1370-1373. 
25. Spinelli, A.; Gerrits, M.; Toskovic, R.; Bryant, B.; Ternes, M.; Otte, A. F. Nat Commun 2015, $6,10046$.

26. Schmidt, A. R.; Hamidian, M. H.; Wahl, P.; Meier, F.; Balatsky, A. V.; Garrett, J. D.; Williams, T. J.; Luke, G. M.; Davis, J. C. Nature 2010, 465, 570-576.

27. Mizukami, Y.; Shishido, H.; Shibauchi, T.; Shimozawa, M.; Yasumoto, S.; Watanabe, D.; Yamashita, M.; Ikeda, H.; Terashima, T.; Kontani, H.; Matsuda, Y. Nat Phys 2011, 7, 849-853.

28. Sahling, S.; Remenyi, G.; Paulsen, C.; Monceau, P.; Saligrama, V.; Marin, C.; Revcolevschi, A.; Regnault, L. P.; Raymond, S.; Lorenzo, J. E. Nat Phys 2015, 11, 255-260.

29. Gunlycke, D.; Jefferson, J. H.; Rejec, T.; Ramsak, A.; Pettifor, D. G.; Briggs, G. A. D. J. Phys. Cond.Mat. 2006, 18, S851.

30. Bazhanov, D. I.; Stepanyuk, O. V.; Farberovich, O. V.; Stepanyuk, V. S. Phys. Rev. B 2016, 93, 035444.

31. Hirjibehedin, C. F.; Lin, C.-Y.; Otte, A. F.; Ternes, M.; Lutz, C. P.; Jones, B. A.; Heinrich, A. J. Science 2007, 317, 1199-1203.

32. Barral, M. A.; Roura-Bas, P.; Llois, A. M.; Aligia, A. A. Phys. Rev. B 2010, 82, 125438.

33. Bryant, B.; Spinelli, A.; Wagenaar, J. J. T.; Gerrits, M.; Otte, A. F. Phys. Rev. Lett. 2013, 111, 127203.

34. Choi, D.-J.; Robles, R.; Gauyacq, J.-P.; Ternes, M.; Loth, S.; Lorente, N. Phys. Rev. B 2016, 94, 085406.

35. Anderson, P. W. Phys. Rev. Lett. 1966, 17, 95.

36. Appelbaum, J. A. Phys. Rev. 1967, 154, 633.

37. Gauyacq, J.-P.; Yaro, S. M.; Cartoixà, X.; Lorente, N. Phys. Rev. Lett. 2013, 110, 087201.

38. Amico, L.; Fazio, R.; Osterloh, A.; Vedral, V. Rev. Mod. Phys. 2008, 80, 517-576.

39. Henderson, L. Vedral, V. J. Phys. A 2001, 34, 6899.

\section{ACKNOWLEDGEMENTS}

D.J.C., J.A.J.B., S.Y., S.R.P. and S.L. acknowledge Edgar Weckert and Helmut Dosch (Deutsches Elektronen-Synchrotron, Hamburg, Germany) for providing high-stability lab space. D.J.C. and J.A.J.B. acknowledge postdoctoral fellowship from the Alexander von Humboldt foundation. M.T. acknowledges support from the DFG-SFB 767. J.A.J.B. acknowledges support from the Natural Sciences and Engineering Research Council of Canada. N.L. and R.R. acknowledge support form Spanish MINECO (Grant No. MAT2012-38318-C03-02 with joint financing by FEDER Funds from the European Union). ICN2 acknowledges support from the Severo Ochoa Program (MINECO, Grant SEV-2013-0295). 
Figure 1:
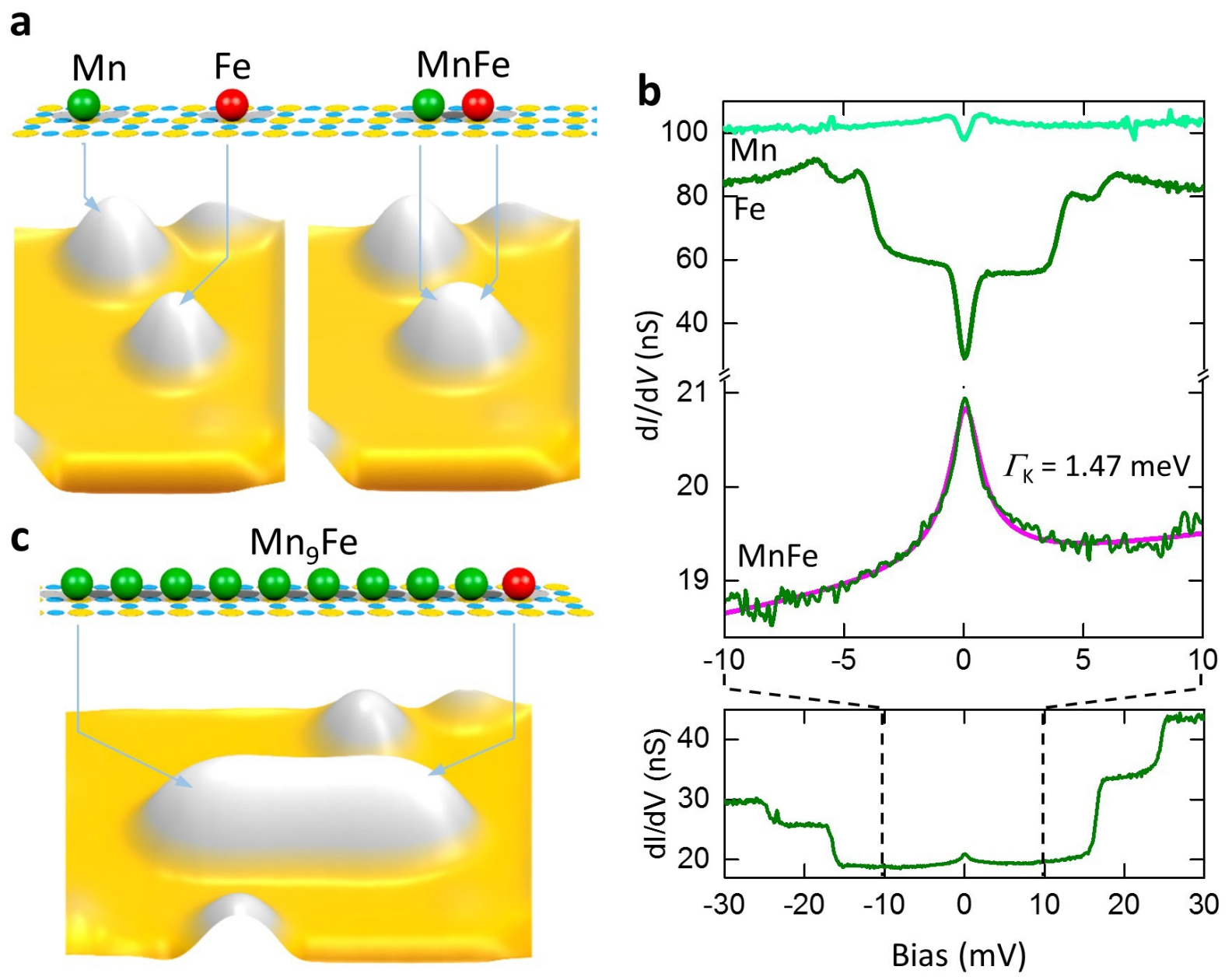

d

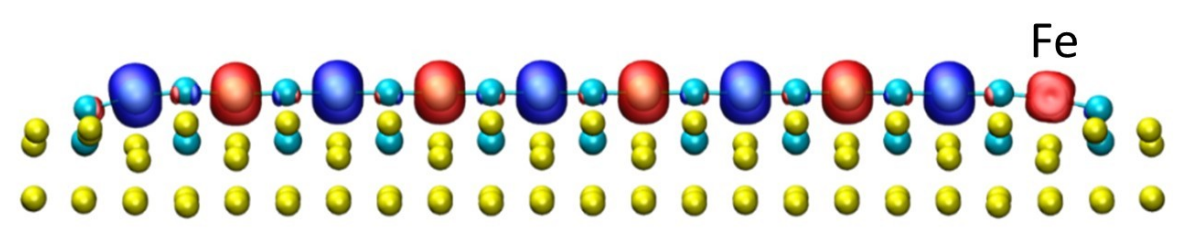


Figure 2:
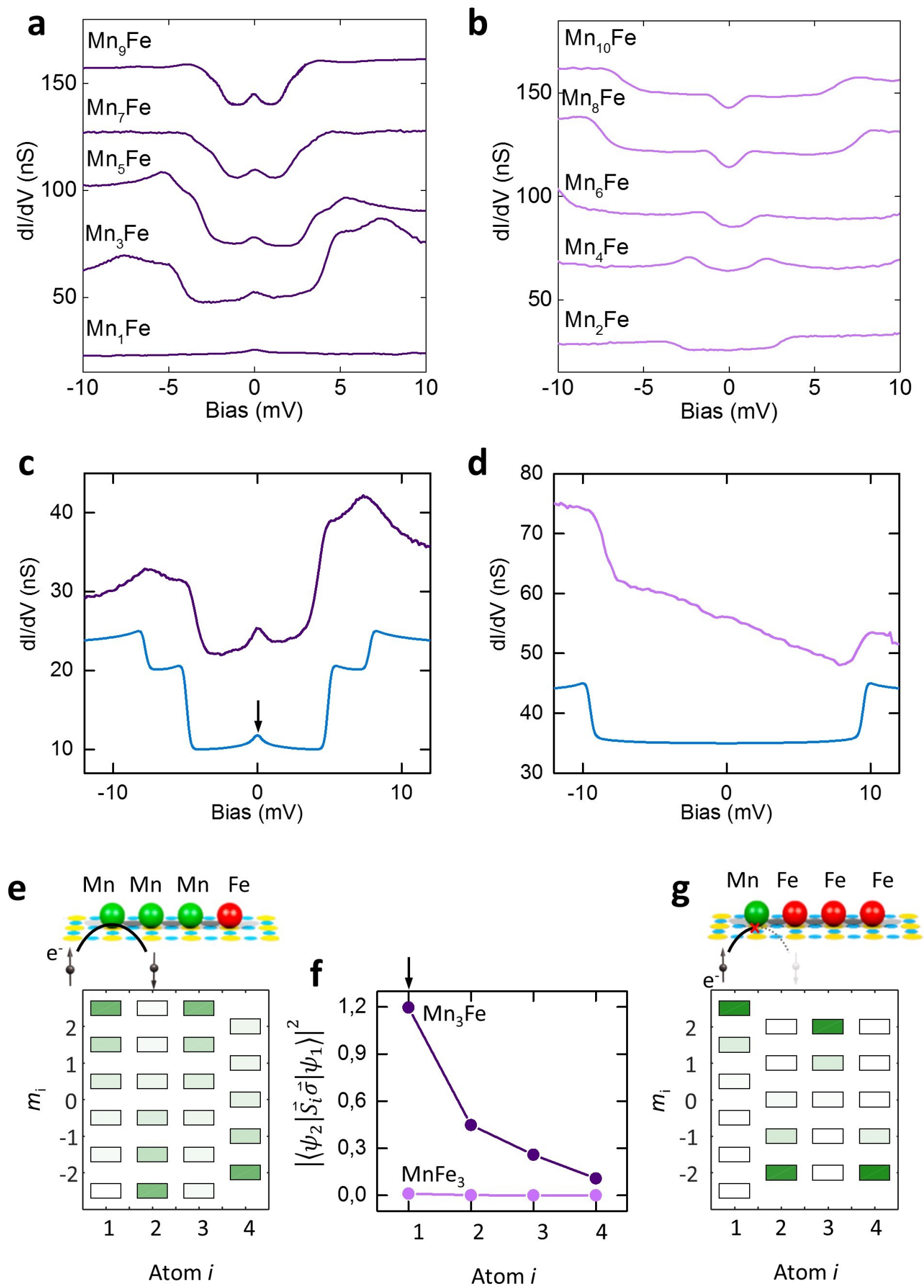
Figure 3:

a

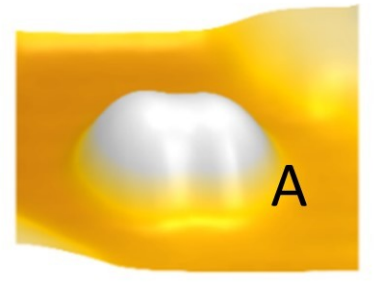

double-spaced

b
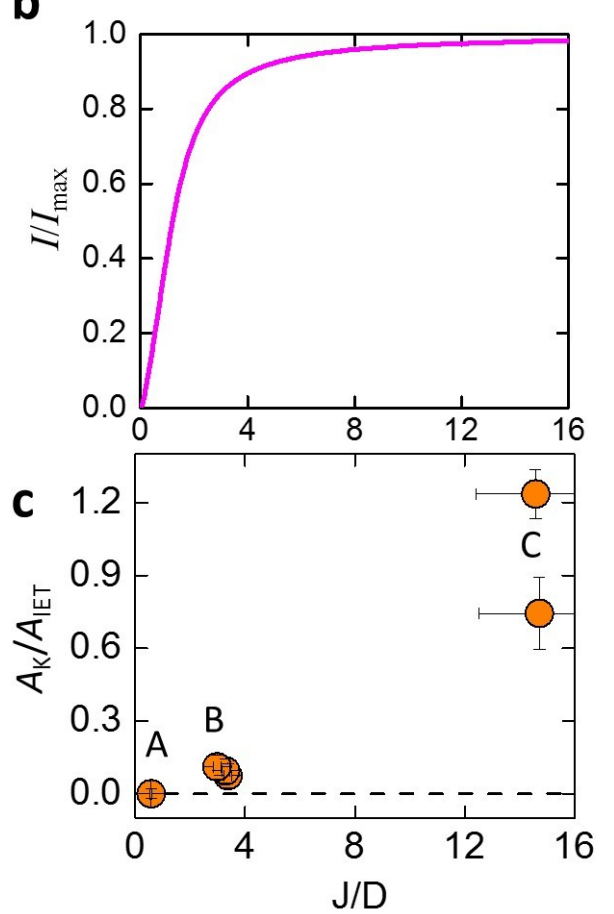

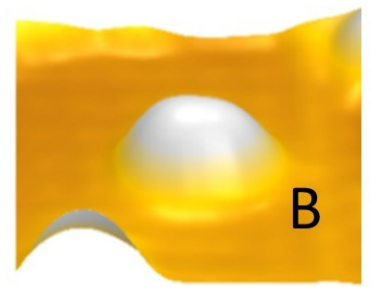

single-spaced
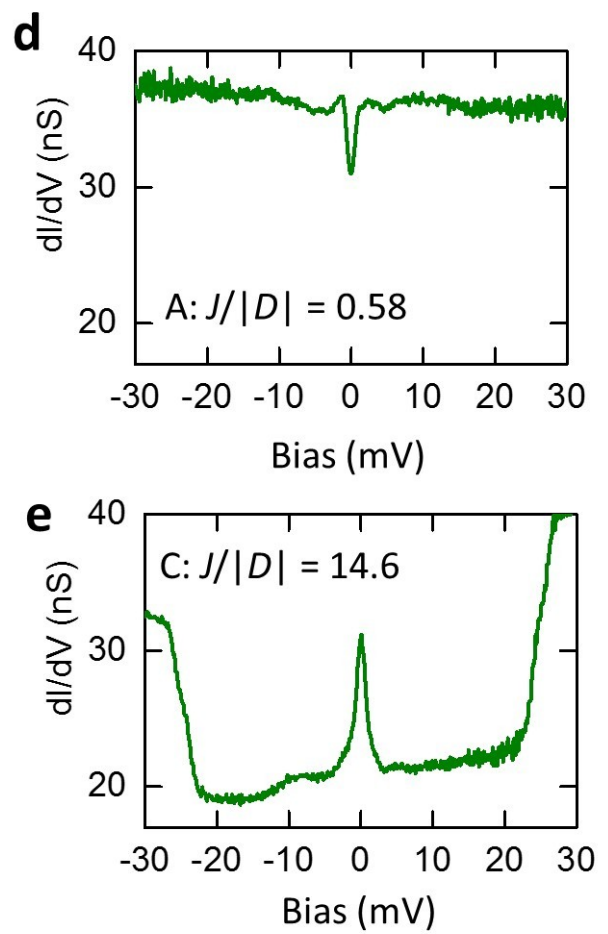

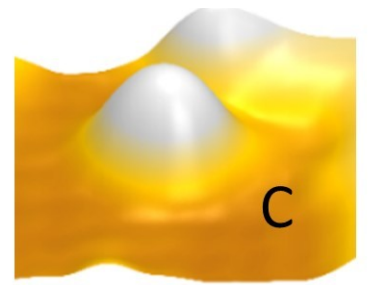

compact
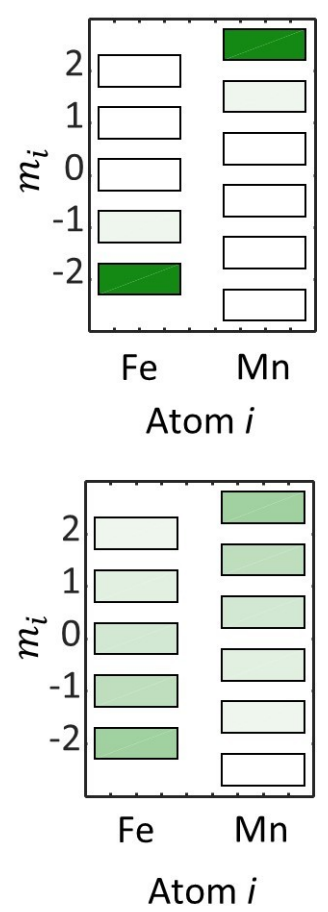
Figure 4:
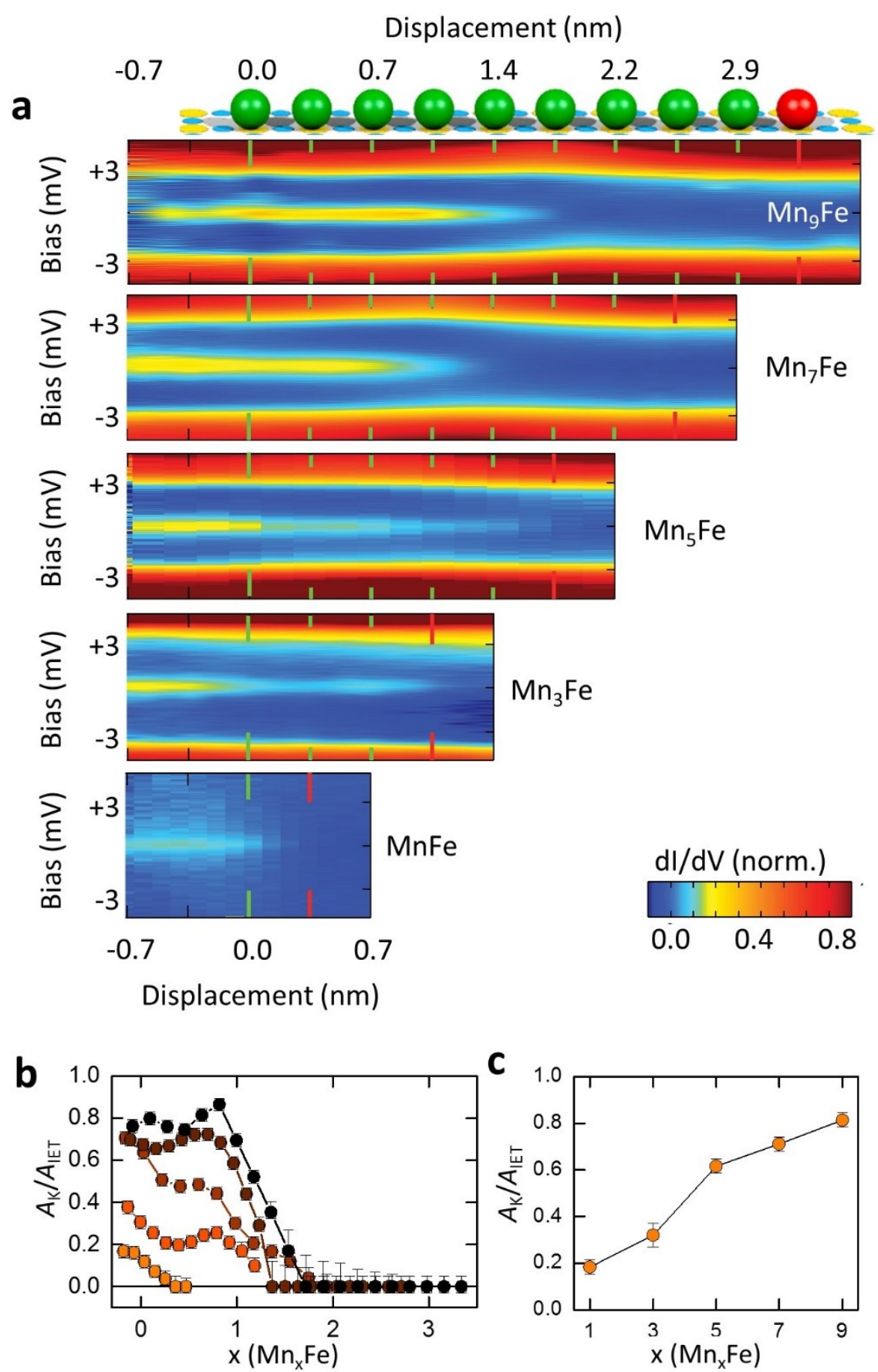